Voix et Images

volxetimages

\title{
Obscénités du roman contemporain
}

\section{Michel Biron}

Volume 24, numéro 3 (72), printemps 1999

La littérature québécoise sous le regard de l'autre

URI : https://id.erudit.org/iderudit/201453ar

DOI : https://doi.org/10.7202/201453ar

Aller au sommaire du numéro

\section{Éditeur(s)}

Université du Québec à Montréal

ISSN

0318-9201 (imprimé)

1705-933X (numérique)

Découvrir la revue

Citer cet article

Biron, M. (1999). Obscénités du roman contemporain. Voix et Images, 24(3),

598-604. https://doi.org/10.7202/201453ar d'utilisation que vous pouvez consulter en ligne.

https://apropos.erudit.org/fr/usagers/politique-dutilisation/ 


\title{
Obscénités du roman contemporain
}

\author{
Michel Biron, Université du Québec à Montréal
}

Rien de plus tentant que d'écrire le roman d'une époque, d'une génération, d'une révolution, de "faire tenir l'océan dans une carafe.", comme le disait Flaubert à propos de son Éducation sentimentale, son roman moderne. Tel est le projet du premier roman de l'essayiste Jean Larose, intitulé Première jeunesse ${ }^{1}$. Mais l'océan n'a pas tout à fait les mêmes dimensions quand on l'aborde depuis Saint-Françoisd'Iberville. La Révolution tranquille n'entretient que de très vagues ressemblances avec 1848 ou avec les grandes révolutions modernes. Si elle résonne des cris et des chants inventés par d'autres jeunesses, celles de Californie ou de Paris, elle les retraduit dans le langage jésuitique du collège classique fréquenté par François, le narrateur. Son éducation 
sentimentale, il la fait en écrivant une pièce de théâtre d'un genre "tragique-moderne" pour marquer la fin de l'année. La révolution tient dans une pièce de théâtre, comme l'océan dans une carafe.

Filée d'un bout à l'autre du roman, la métaphore théâtrale transforme les désirs et les sentiments individuels en désirs et sentiments spectaculaires. Le corps et le langage, tournés entièrement vers l'extérieur, sont offerts au regard collectif, comme si le seul fait d'exhiber son désir constituait une inépuisable transgression. La fin et les moyens sont identiques: je me thêâtralise, je fais du monde un thêâtre, sans autre motivation que l'acte lui-même. Le théâtre occupe la place du réel, sans l'abolir pour autant. Ce qu'il abolit avec allégresse, ce sont les frontières séparant l'intériorité et l'extériorité, le public et la scène, le sujet individuel et le sujet collectif. Ces frontières, il est vrai, étaient mal gardées. Le "moinous " des jeunes ne rencontre aucune résistance sérieuse et s'amuse à être le plus "grave" possible, comme si c'était un jeu davantage qu'un combat. Son pouvoir de transgression ne heurte que mollement les "autorités ": dans le Québec de la Révolution tranquille, les figures du père ou du professeur sont si faibles qu'elles n'offrent aucune résistance sérieuse. Le collège classique, institution emblématique de "l'Ancien Régime", s'effondre presque de luimême, comme si les bases sur lesquelles il reposait étaient faites de sable. François éprouve même une sorte de pitié sincère pour ces êtres soudainement anachroniques, acteurs pressés de changer de rôle et qui ne trouveront rien de mieux que de jeter la soutane afin d'exhiber, eux aussi, leur "sens de l'histoire".

À cette impudeur généralisée, nulle n'est mieux préparée que Solange, l'ancienne de François, véritable déesse du sexe qui a compris que la véritable obscénité consiste à dévoiler non pas le corps nu, mais la "nudité de sens":

\begin{abstract}
Attentive par-dessus tout au choc que la scène devait infliger aux spectateurs - au-delà de nos amis réunis, à la cité entière qu'ils étaient chargés de représenter, comme le chour dans les tragédies -, la petite géniale avait senti que, mieux que l'obscénité, le rythme aspirant et foulant de sa caresse avait une nudité de sens et un vide d'intention scandaleux. (p. 39)
\end{abstract}

Mais scandaleux pour qui? François, il est vrai, n'apprécie pas trop de voir son amoureuse d'hier pratiquer une fellation publique sur Aurélien, son meilleur ami, mais on ne lui demande pas son avis. Il est saisi par le spectacle - comment ne pas l'être? - , pourtant il s'y laisse prendre plutôt qu'il n'y participe. Il ne cherche tout simplement pas à s'y soustraire, souffrant avec un plaisir presque masochiste de l'exclusion dont il est l'objet et s'abandonnant sans enthousiasme au vertige du happening. La révolution est vécue par lui comme une peine d'amour inavouée, c'est-à-dire comme quelque chose de déjà fini.

D'où l'ambiguïté qui traverse tout le roman: la révolution est tantôt une farce, comme le disait Marx à propos des périodes où l'Histoire se répète, tantôt un échec personnel, à la façon d'un rendez-vous manqué qui ne cesse de nous hanter. C'est surtout le premier aspect qui ressort 
à travers les figures radicales de Solange et d'Aurélien, sans parler de la galerie de personnages secondaires qui sont les véritables bouffons involontaires de l'époque, par exemple l'optométriste Ferguson ou le notaire Couturier. Ce sont les pharmaciens Homais du roman, affublés d'un style hautement comique, toujours "ouverts" aux jeunes et d'autant plus ridicules. Ils s'expriment au moyen de lieux communs et de proverbes le plus souvent déformés, comme si le langage le plus convenu était lui-même une pâte molle, dépourvue de structure et d'histoire: "C'est très cela! Dans la vie, faut savoir de quel côté de la tranche que ton pain est beurré! Sinon, tu risques de te faire poigner ventre à terre les culotte baissées". Ou encore: "Le temps est peut-être venu de brasser la baraque. Gerry me dit que toi et tes amis, vous êtes ben contestataires. Je suis pas contre, au contraire je suis pour à cent pour cent. Faitesnous une belle pièce de contestation." (p. 185)

Devant ce conformisme de la révolte, François ne sait trop comment (ré)agir. Au fur et à mesure qu'il cherche à prendre ses distances, Aurélien se moque de lui et de ses prétentions artistiques. Il est pour le "théâtre vrai", celui de la vie, de l'immédiateté. François commence, au contraire, à creuser la fosse entre la scène et la salle - et, du même coup, entre Aurélien et lui. Il ne croit plus en Aurélien, ni en Solange, ni à la révolution, ni même à sa propre pièce de théâtre. Il veut "faire de la scène une tour d'ivoire" ( $\mathrm{p}$. 162), c'est-à-dire, à la limite, congédier le spectateur afin de sortir enfin de la tyrannie du spectaculaire: Mais il est jeune, lui aussi, et le conflit esthétique ne l'intéresse que moyennement, pas plus que les autres conflits. Il veut écrire, certes, mais pas à n'importe quel prix. De toute façon, sous la pression des parents, la pièce est finalement annulée par le directeur général. Il y a bien une occupation spontanée des lieux par les collégiens regroupés autour d'une Assemblée générale révolutionnaire, mais elle ne dure que quelques jours. Puis, rien. François renonce de lui-même au théâtre, préférant la philosophie. Aurélien choisit de se concentrer sur le rythme de sa respiration, "imbécile installé dans la sagesse éternelle" (p. 216), selon l'expression peu amicale de François. Ils n'ont plus rien en commun et François s'en retourne chez ses parents.

Adieu la révolution, voici la famille, les tartines au MapOSpread du frère, les colères impuissantes du père, l'irrésistible et quasi incestueux amour maternel. Difficile d'y trouver un climat propice à la révolte poétique. Le voudrait-il qu'il serait déjà trop tard: le collège n'existe plus, rasé par un incendie spectaculaire, comme pour servir de scène finale au "théâtre vrai" d'Aurélien. François se retourne alors contre ce monde nouveau qui prend plaisir à voir brûler les pyramides avec les musées et il se jure, à l'instar de Rimbaud travaillant à s'encrapuler, de devenir détestable. Il accuse: "Tout ça, c'est de la faute à ces tarlats de dramaturges qui ne supportent pas la séparation de l'écrivain et du peuple." (p. 287) Suit une liste d'insultes adressées aux écrivains de la rue Fabre, dramaturges et romanciers confondus. Seuls les poètes de la Révolution tranquille échappent au 
réquisitoire. Pourquoi tout à coup une telle remontée de la colère? On ne le sait trop et on ne comprend pas davantage contre qui elle est dirigée si l'on ne suit de près l'actualité littéraire locale, si l'on ne possède d'avance les clés de ce petit hermétisme. La voix de l'essayiste ou du pamphlétaire se hausse et enterre la voix du romancier, qui a toutes les peines du monde à reprendre le fil de son histoire et à se faire entendre. Ce fil est cassé par une sorte de pudeur insurmontable, suprenante dans un roman si impudique par ailleurs, une pudeur d'écrivain qui, selon ses propres mots, lui "interdit de raconter" (p. 297).

Ainsi s'achève le roman-essai de Larose, comme s'il constatait à la fin l'échec, voire l'impossibilité du projet romanesque. De même que la pièce de François, le roman n'a pas vraiment lieu. Les idées, les intentions limitent la singularité des personnages au point de les figer dans leur rôle. Des pages superbes, il n'en manque pas dans Première jeunesse, mais ce sont des pages, justement. Sur la scène romanesque proprement dite, Solange, Aurélien et les autres sont des personnages faussement vraisemblables, trop typiques, trop représentatifs, trop fabriqués. Des clones du pharmacien Homais, avec des proverbes plein la bouche, sans dessus ni dessous.

\section{**}

L'aveu final du narrateur de Première jeunesse, sa gêne de raconter (le verbe est intransitif chez Larose), pointe vers une difficulté plus générale du roman actuel. La principale fonction de celui-ci n'est peut-être plus, comme ce fut le cas pendant un siècle au moins, de raconter. Le roman aurait plutôt pour tâche première de témoigner. C'est du moins ainsi que se présentent de nombreux romans contemporains, comme La danse juive de Lise Tremblay $^{2}$. Il fut un temps où l'obésité de la narratrice de ce roman aurait été une simple caractéristique physique permettant au lecteur d'accorder ou non de la vraisemblance aux actions du personnage. Mais la narratrice de La danse juive n'est au centre d'aucune aventure en dehors de sa vie. Pour paraphraser une formule célèbre de Jean Ricardou, nous ne lisons plus l'aventure d'une femme obèse, mais l'obésité comme aventure, comme l'aventure de toute une vie ${ }^{3}$. Puisqu'il y a malgré tout du récit, on peut bien continuer de parler de "roman", mais le romanesque a été absorbé et recyclé par une autre forme de récit, que nul terme ne désigne mieux, semble-t-il, que celui de témoignage. Certes, cela n'est pas nouveau: la littérature de témoignage est peut-être au $\mathrm{xx}^{\mathrm{e}}$ siècle ce que le roman réaliste fut au XIX ${ }^{e}$. Ses formes sont multiples, du récit de soi issu de la psychanalyse au reportage journalistique (témoignage direct de l'actualité) en passant par les livres et les films de témoignage associés à la guerre et, plus récemment, au sida. La nouveauté vient peut-être du fait que le récit de témoignage se détache aujourd'hui de l'événement ou, plus exactement, qu'il peut se passer de l'événement (d'une cause, dans tous les sens du mot), qu'il tire sa légitimité de cela même qui le rend obscène.

La littérature de témoignage, suggérait le critique Ross Chambers 
dans une conférence prononcée il y a deux ans à Montréal, constitue, au sens fort, une "pratique culturelle". Elle ne se construit pas à la façon d'un genre précis et ne cherche pas à transgresser les limites des genres existants comme l'a fait durant quelques années le Nouveau Roman. Elle détourne les genres dont dispose l'écrivain ou l'artiste et donne à voir des objets qui n'appartiennent pas à l'ordre de la culture. C'est une littérature désœuvrée qui a pour caractéristique de mettre à l'avant-scène ce qui d'ordinaire se trouve derrière les rideaux - d'où, au sens littéral, son obscénité. Elle tend à privilégier ce dont on ne peut pas parler (la mort) et ce dont on ne veut pas parler (le tabou). Le paradoxe, c'est qu'en faisant du tabou une sorte de thème obligé elle joue sans cesse "sur nos nerfs", sur notre fibre la plus sensible. Elle produit ainsi un effet de hantise sur l'ensemble de la sphère culturelle, comme si elle nous forçait à consentir, dans notre pratique de la culture, à ce qui là déborde. C'est une littérature non plus de l'imitation, de la mimésis, mais du surplus.

Le surplus, dans La danse juive, crève les yeux: le corps énorme et littéralement insupportable de la narratrice a quelque chose d'intrinsèquement obscène. Ce corps la trahit à chaque geste, lui coûte mille peines, mille douleurs. Il fait mal à voir et elle ne se prive pas de s'en servir justement pour punir ceux qui l'ont créée ainsi, monstre médiocre. Il faut voir comment elle se débarrasse brusquement de son amie Alice, qui dit "mature" pour qualifier son dernier amant et qui voudrait tant faire partie du "milieu". Même mépris ostensible vis-à-vis de sa mère, qui n'a pas le courage de la regarder ailleurs que dans les yeux. Tout cela, conclut-elle, c'est la faute du père, de qui lui vient son obésité maladive les femmes dans la famille paternelle sont toutes grosses. Il en paiera le prix à la fin, le pauvre homme, la gorge tranchée par sa fille. Le lecteur éprouve alors une soudaine sympathie pour la meurtrière, sans trop savoir pourquoi elle est la plus à plaindre dans cet univers grazévisqueux. Peut-être est-il simplement soulagé de la voir enfin "passer à l'acte"? Peut-être est-il rassuré de toucher in extremis à l'intériorité jusque-là insaisissable du personnage? Peut-être ce geste extrême n'est-il là, après tout, que pour indiquer au lecteur que le livre entre ses mains est bel et bien un roman?

Reste qu'on se demande jusqu'à la fin: qu'est-ce que La danse juive? Il est question de danse (la narratrice joue du piano dans une école de danse) et l'un des personnages (Mel, l'amant de la narratrice) est juif. Mais il n'est question de danse juive qu'à un moment, très bref, alors que la narratrice aperçoit un tableau montrant des Juifs hassidiques en train de danser. Cette façon de transformer un détail en emblème est toutefois typique de la manière d'écrire de Lise Tremblay, qui remet le superficiel au premier plan. La violence de cette écriture tient peut-être justement dans le refus de la profondeur. La phrase glisse, brutale, sèche, exacte. Rien d'exagéré, rien de saillant, pas même le malheur de la narratrice. De temps en temps, il lui arrive de rire franchement (avec Paul, son ami musicien). Quelquefois, mais de façon très furtive et sans plaisir, elle fait l'amour (avec Mel). Ce sont des 
instants qu'elle ne cherche nullement à prolonger. Le bonheur ne l'intéresse pas. "Je ne voulais pas être sauvée" (p. 67), dira-t-elle en mangeant des chocolats au fond de l'autobus. L'avenir, l'âme, les grands sentiments, les sensations fortes, la passion, la révolte, tout cela est enfoui dans la graisse du personnage. Invisible.

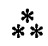

Le cimetière des éléphants, d'Hélène Rioux ${ }^{4}$, désigne en fait une de ces îles où les gens vont chercher le soleil. À première vue, le paradis habituel: le climat est sain, la mer est bleue, la vie est facile, les gens ne manquent de rien. La narratrice y est venue pour écrire en paix un livre sur l'histoire des sectes et des mouvements d'extrême droite en Amérique du Nord. Elle s'intéresse aux groupes et ne croyait pas si bien tomber en choisissant de séjourner, hors-saison, parmi le "troupeau" de retraités, "tous plus ou moins paumés, nostalgiques, éclopés, malmenés par l'amour, rejetés par les flots, échoués comme des épaves que la mer abandonne sur le rivage" (p. 28). Il ne s'agit pas à proprement parler d'une secte, mais cette société sans enfants ni pauvres n'est pas dépourvue d'organisation, de rituels. En plus des quelques cafés où les gens se rencontrent assez régulièrement, on y trouve un club social et une Amicale des résidents qui multiplie les activités de groupe.

La narratrice pratique ici un genre de plus en plus important dans la littérature mondiale actuelle: le tourisme politique. Nous ne sommes bien sûr ni en Croatie ni même à
Cuba, mais le principe est semblable, la violence en moins. Elle ne se contente pas de profiter des lieux en restant à distance des gens qui les habitent: elle se mêle à eux et recueille leur histoire, leur témoignage. Le roman se construit ainsi comme une collection de portraits plus ou moins détaillés, grâce auxquels se forme l'image d'une société hétérogène, composée de gens venus d'un peu partout pour travailler, pour vieillir, peut-être pour mourir sous des cieux plus cléments. Malgré la connotation grégaire du titre, ce sont les individus qui retiennent toute son attention. Elle les écoute avec une attention dénuée de jugement, étonnée de trouver là, dans un milieu aussi protégé, les mêmes conflits, les mêmes passions, les mêmes malheurs que dans sa propre société.

La banalité n'a rien pour effrayer la narratrice, qui commence sa série de portraits par celui d'Hubert, homme dépressif qui s'est passionné pour une jeune fille, Miranda. Une nuit, alors que Miranda a disparu, Hubert se met à raconter ses malheurs à la narratrice en espérant on ne sait trop quoi. Il n'avait guère de choses à cacher, il n'en a plus du tout. Le caché est transparent, si visible, si prévisible qu'il perd le peu de charme qu'on aurait aimé lui accorder. Aucune ombre, aucun secret, aucun mystère: l'égocentrisme et l'impudeur sont tout un. Une fois l'homme percé de bout en bout, il peut retourner à la vie: on apprendra plus tard qu'il a définitivement jeté Miranda à la rue et qu'il s'est trouvé une nouvelle maîtresse.

Puis il y a Renata, la Napolitaine, célibataire dans la cinquantaine après au moins trois mariages. Magali, la 
patronne du café Mimosa où va régulièrement la narratrice. La vieille Olga, dont la mort plonge Boris dans le désespoir. Michel-Ange, peintre paysagiste pas tout à fait sain d'esprit, qui finira par se jeter dans la mer. Un Américain sadique propriétaire d'une vaste maison sur le flanc de la colline, en marge du village et de la société. Et une douzaine d'autres esquisses de portraits, parfois réduits à un seul paragraphe. Tous ces gens, plus ou moins volontairement coupés de leur passé, promènent leur mémoire en attendant de mourir. Il n'y a pas de mémoire collective pour unifier ces histoires de vie : pas de récit commun. Ils sont seuls et tristes à regarder, tantôt insignifiants avec leur manie d'organiser des parties de boules et des cassoulets, tantôt irréels comme Michel-Ange. L'humanité vieillissante dans un club social, comme l'océan dans une carafe.

1. Jean Larose, Première jeunesse, Montréal, Leméac, 1998, 307 p.

2. Lise Tremblay, La danse juive, Montréal, Leméac, 1999, 143 p.

3. La formule originale était: * Le roman n'est plus l'écriture d'une aventure mais l'aventure d'une écriture. *

4. Hélène Rioux, Le cimetière des éléphants, Montréal, XYZ éditeur, 1998, 187 p. 